\title{
Joint Power Allocation and Beamforming in Amplify-and-Forward Relay Networks under Per-Node Power Constraint
}

\author{
Farzin Azami, Seyed Mostafa Safavi Hemami, and Abbas Akbarpour-Kasgari
}

Department of Electrical Engineering, Amirkabir University of Technology (Polytechnic), Tehran, Iran

Correspondence should be addressed to Seyed Mostafa Safavi Hemami; msafavi@aut.ac.ir

Received 30 September 2016; Revised 7 February 2017; Accepted 5 March 2017; Published 28 May 2017

Academic Editor: Gonzalo Vazquez-Vilar

Copyright (C) 2017 Farzin Azami et al. This is an open access article distributed under the Creative Commons Attribution License, which permits unrestricted use, distribution, and reproduction in any medium, provided the original work is properly cited.

\begin{abstract}
Two-way relay networks (TWRN) have been intensively investigated over the past decade due to their ability to enhance the performance assessment of networks in terms of cellular coverage and spectral efficiency. Yet, power control in such systems is a nontrivial issue, particularly in multirelay networks where relays are deployed to ensure a required Quality of Service (QoS). In this paper, we envision to address this critical issue by minimizing the sum-power with respect to per-node power consumption and acceptable users' rates. To tackle this, we employ a variable transformation to turn the fractional quadratically constrained quadratic problem (QCQP) into semidefinite programming (SDP). This algorithm is also extended to a distributed format. Simulation results of deploying 10 relay stations reveal that the total power consumption will decrease to approximately $8 \mathrm{dBW}$ for $6 \mathrm{bps} / \mathrm{Hz}$ sum-rate.
\end{abstract}

\section{Introduction}

Cooperative communications $[1,2]$ have received a great attention of research interest as a promising way to capture virtual diversity. Also, relay networks can provide an extended coverage and enhance the spectral efficiency, especially at the edges of cells $[3,4]$. Hence, a considerable number of papers evaluated power control of such networks due to their complexity and importance, particularly in two-way relay networks (TWRN) [5-7]. In this light, there are also further research efforts that minimize the sum-power while each user enjoys a minimum required signal-to-noise ratio (SNR) as its Quality of Service (QoS) [8].

Recently, the joint optimization of the power allocation and bidirectional network distributed beamforming is studied in [9-11]. These works explore the optimality of jointly relay beamforming and transceivers' power. Precisely speaking, the minimum total transmit power and max-min fair design criterion are used in these works under QoS and total transmit power constraints, respectively. Besides, semiclosed-forms are also represented in these works. They also address the joint relay selection and power allocation with respect to total power constraint. In [12], the achievable rate region is obtained in the aforementioned scenario. Authors in [13] maximize the sum-rate under total relay's power constraint via two-way relay beamforming.

Prior works in this context enforce the sum-power constraint on all nodes of network $[5,6]$. However, in practice, having only one unit source to supply all nodes imposes some formidable challenges due to the distance among the users. Inspired by the above discussion, the best suitable alternative might be a distributed power constraint on each node [8].

The problem of power control can be formulated as a fractional quadratically constrained quadratic problem (QCQP). It is shown in [14, 15] that Charnes-Cooper [16] transformation can relax the fractional QCQP to semidefinite programming (SDP), and the optimal solution is given by using suitable rank-one transformation [16]. However, we have used the preliminary solution in optimizing the power control context.

In this paper, we have proposed a beamforming and power allocation algorithm based on minimizing the power with respect to available end-user rates and individual power constraints. This is a case of practical interest and to the best of our knowledge there is no prior work to address these considerations together. In this system model, we presume 


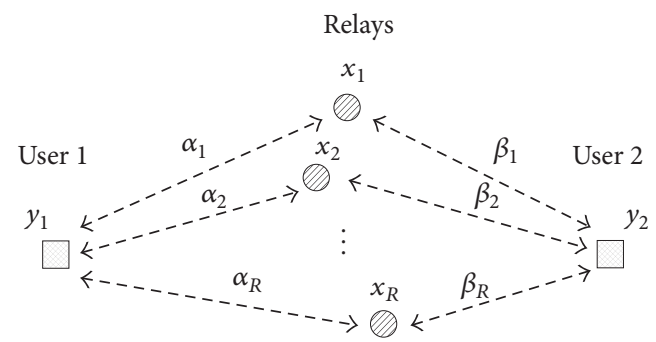

FIgURE 1: Multirelay communication network.

that there exist a control center which is fully aware of global channel state information (CSI), while only local CSI is available on each node. More precisely, each relay node estimates gains of its own channels which are shared with two users. After that, all relay nodes send their channels information to the control center. A power allocation framework is used to model the problem, solved through convex programming method. This method uses Charnes-Cooper transformation and SDP relaxation to the optimization problem. Furthermore, we considered this problem constrained to a limited source of energy for each node in TWRN, as a practical assumption. A distributed version of the proposed algorithm was represented which determines each of the weights and power individually by only using some common parameters. Besides, common parameters were determined and transmitted by a control center in the network. Moreover, to show effectiveness of the proposed method, we introduced an efficiency metric that uses sum-rate to the consumed sumpower. This metric will grow in value when the number of relays increases.

The remainder of the paper is organized as follows. In the next section, we describe the system model and problem formulation followed by our proposed algorithm in Section 3. Section 4 delineates the distributed implementation of the beamforming and power determination algorithm. Simulation results and conclusion are presented in Sections 5 and 6, respectively.

Notations. For a matrix $\mathbf{A}, \mathbf{A}^{T}$ and $\mathbf{A}^{H}$ denote the transpose and transpose-conjugate of $\mathbf{A}$, respectively. For a complex scalar $a,|a|$ indicates its magnitude and $\measuredangle a$ exhibits its angle. For a vector a, $\|\mathbf{a}\|$ represents its Euclidean norm. Moreover, $\mathbf{D}(\mathbf{a})$ denotes a diagonal square matrix with vector a along its main diagonal. For two vectors $\mathbf{a}$ and $\mathbf{b}, \mathbf{a} \otimes \mathbf{b}$ represents $\mathbf{a D}(\mathbf{b})$.

\section{System Model}

Consider a multirelay network which includes two transceivers and $R$ amplify-and-forward (AF) relay nodes, where bidirectional communication links are accomplished deploying a two-way relay scheme depicted in Figure 1. Users send their data stream through a narrow band flat fading propagation channel [8]. Let $\boldsymbol{\alpha} \in \mathbb{C}^{R \times 1}$ denote the channel coefficient vector between user 1 and relays, and $\boldsymbol{\beta} \in \mathbb{C}^{R \times 1}$ denotes the channel coefficient vector between user 2 and relays. In addition, $\boldsymbol{\alpha}$ and $\boldsymbol{\beta}$ are assumed to be known at the relay stations. We also assume that transmission power by each user does not exceed $\delta_{1}$ and $\delta_{2}$, respectively, and the power of $i$ th relay is upper limited by $\rho_{i}$ for $i=1,2, \ldots, R$. We assume there exists no direct link among the users due to the heavy shadowing and path loss phenomenon. In this light, we conclude that the two-hop network is rate-optimal [7]. Within each coherence interval, the information exchange occurs in two phases: the multiple access phase and the broadcast phase.

In other words, in the first time slot, the transceivers simultaneously broadcast their data to all relays, and in the second time slot, relays forward the amplified and phase tilted version of the received signals to destinations. During the multiple access phase, both transceivers send their normalized symbols, $s_{1}$ and $s_{2}$, to relay nodes, simultaneously. The received signal at the relays can be expressed as

$$
\mathbf{x}=\sqrt{P_{1}} \boldsymbol{\alpha} s_{1}+\sqrt{P_{2}} \beta s_{2}+\mathbf{z}
$$

where $\mathbf{x} \in \mathbb{C}^{R \times 1}$ is the vector of received signal at the relays and $P_{1}$ and $P_{2}$ are transmit powers of user nodes, respectively, while the $R$-dimensional vector $\mathbf{z}$ models the zero-mean additive complex Gaussian noise with unit variance. Besides, $\boldsymbol{\alpha}=\left[\alpha_{1}, \alpha_{2}, \ldots, \alpha_{R}\right]^{T}$ and $\boldsymbol{\beta}=\left[\beta_{1}, \beta_{2}, \ldots, \beta_{R}\right]^{T}$ are the channel coefficients vectors. In the broadcast phase, the relays transmit the tilted amplified version of the received signal to the users. Let the $R \times 1$ complex vector $\mathbf{t}$ as the broadcast signal of all relay nodes, and $\boldsymbol{\omega}=\left[\omega_{1}, \omega_{2}, \ldots, \omega_{R}\right]^{T}$ as the amplification factor of relay nodes, then we can retrieve the broadcast signal as

$$
\mathbf{t}=\mathbf{x} \otimes \boldsymbol{\omega}
$$

Thus, the received signals at two user nodes can be expressed as

$$
\begin{aligned}
\tilde{y}_{1} & =\boldsymbol{\alpha}^{T} \mathbf{W} \mathbf{x}+n_{1} \\
& =\boldsymbol{\alpha}^{T} \mathbf{W}\left(\sqrt{P_{1}} \boldsymbol{\alpha} s_{1}+\sqrt{P_{2}} \boldsymbol{\beta} s_{2}+\mathbf{z}\right)+n_{1}, \\
\tilde{y}_{2} & =\boldsymbol{\beta}^{T} \mathbf{W} \mathbf{x}+n_{2} \\
& =\boldsymbol{\beta}^{T} \mathbf{W}\left(\sqrt{P_{1}} \boldsymbol{\alpha} s_{1}+\sqrt{P_{2}} \boldsymbol{\beta} s_{2}+\mathbf{z}\right)+n_{2},
\end{aligned}
$$

where $\mathbf{W}=\mathbf{D}(\boldsymbol{\omega})$ represents the diagonal matrix with $\boldsymbol{\omega}$ as its main diagonal and $n_{1}$ and $n_{2}$ are Gaussian distributed noises with zero mean and unit variance. After a simple manipulation, we can restate the received signals in the following way:

$$
\begin{aligned}
\tilde{y}_{1}=\underbrace{\sqrt{P_{1}} \boldsymbol{\omega}^{T} \mathbf{D}(\boldsymbol{\alpha}) \boldsymbol{\alpha} s_{1}}_{\text {self interference }}+\underbrace{\sqrt{P_{2}} \boldsymbol{\omega}^{T} \mathbf{D}(\boldsymbol{\alpha}) \boldsymbol{\beta} s_{2}}_{\text {desired signal }} \\
+\underbrace{\boldsymbol{\omega}^{T} \mathbf{D}(\boldsymbol{\alpha}) \mathbf{z}+n_{1}}_{\text {noise }},
\end{aligned}
$$




$$
\begin{aligned}
\tilde{y}_{2}= & \underbrace{\sqrt{P_{1}} \boldsymbol{\omega}^{T} \mathbf{D}(\boldsymbol{\beta}) \boldsymbol{\alpha} s_{1}}_{\text {desired signal }}+\underbrace{\sqrt{P_{2}} \boldsymbol{\omega}^{T} \mathbf{D}(\boldsymbol{\beta}) \boldsymbol{\beta} s_{2}}_{\text {self interference }} \\
& +\underbrace{\boldsymbol{\omega}^{T} \mathbf{D}(\boldsymbol{\beta}) \mathbf{z}+n_{2}}_{\text {noise }} .
\end{aligned}
$$

As represented, the received signal at both transceivers consists of three different components, namely, self-interference, desired signal, and noise. Since the users are aware of CSI, they can eliminate self-interference corruption and obtain a signal with only noise as its corruption. Hence, the received signals after self-interference cancellation are given as

$$
\begin{aligned}
& y_{1}=\sqrt{P_{2}} \boldsymbol{\omega}^{T} \mathbf{D}(\boldsymbol{\alpha}) \boldsymbol{\beta} s_{2}+\boldsymbol{\omega}^{T} \mathbf{D}(\boldsymbol{\alpha}) \mathbf{z}+n_{1}, \\
& y_{2}=\sqrt{P_{1}} \boldsymbol{\omega}^{T} \mathbf{D}(\boldsymbol{\beta}) \boldsymbol{\alpha} s_{1}+\boldsymbol{\omega}^{T} \mathbf{D}(\boldsymbol{\beta}) \mathbf{z}+n_{2} .
\end{aligned}
$$

According to the signal model, we can define the received SNRs at two terminals as

$$
\begin{aligned}
& \mathrm{SNR}_{1}=\frac{P_{2} \boldsymbol{\omega}^{H} \mathbf{h} \mathbf{h}^{H} \boldsymbol{\omega}}{1+\boldsymbol{\omega}^{H} \mathbf{A} \boldsymbol{\omega}}, \\
& \mathrm{SNR}_{2}=\frac{P_{1} \boldsymbol{\omega}^{H} \mathbf{h} \mathbf{h}^{H} \boldsymbol{\omega}}{1+\boldsymbol{\omega}^{H} \mathbf{B} \boldsymbol{\omega}},
\end{aligned}
$$

where $\mathbf{h}=\left[h_{1}, h_{2}, \ldots, h_{R}\right]^{T}=\boldsymbol{\alpha} \otimes \boldsymbol{\beta}, \mathbf{A}=\mathbf{D}^{H}(\boldsymbol{\alpha}) \mathbf{D}(\boldsymbol{\alpha})>\mathbf{0}$, and $\mathbf{B}=\mathbf{D}^{H}(\boldsymbol{\beta}) \mathbf{D}(\boldsymbol{\beta})>\mathbf{0}$.

Considering $\mathrm{SNR}_{1}$ in (6), the numerator and denominator of $\mathrm{SNR}_{1}$ can be reformulated as

$$
\begin{aligned}
P_{2} \boldsymbol{\omega}^{H} \mathbf{h} \mathbf{h}^{H} \boldsymbol{\omega} & =P_{2}\left|\boldsymbol{\omega}^{H} \mathbf{h}\right|^{2}=P_{2}\left|\sum_{r=1}^{R} \omega_{r}^{*} h_{r}\right|^{2} \leq \sum_{r=1}^{R}\left|\omega_{r}^{*} h_{r}\right|^{2}, \\
1+\boldsymbol{\omega}^{H} \mathbf{A} \boldsymbol{\omega} & =1+\sum_{r=1}^{R}\left|\omega_{r}\right|^{2}\left|\alpha_{r}\right|^{2} .
\end{aligned}
$$

Obviously, the phase of $\boldsymbol{\omega}$ is crucial just in the numerator of $\mathrm{SNR}_{l}$ where according to triangle inequality, equality holds when $\measuredangle \omega_{r}=-\measuredangle h_{r}$. Consequently, having full CSI, the relay coefficients should satisfy $\measuredangle \omega_{r}^{*}=\alpha_{r}^{*} \beta_{r}^{*} /\left|\alpha_{r} \beta_{r}\right|$. The same result can be similarly deduced for $\mathrm{SNR}_{2}$. Hence, from now on in this paper, we assume that $\measuredangle \omega_{r}=-\measuredangle \alpha_{r}-\measuredangle \beta_{r}$, and we bypass the impact of phase distortion. Thus, all the vectors hereafter are absolute values. Specifically, $\boldsymbol{\omega}=\overline{\boldsymbol{\omega}} e^{j \boldsymbol{\theta}}$ and $\overline{\boldsymbol{\omega}}=\left[\bar{\omega}_{1}, \bar{\omega}_{2}, \ldots, \bar{\omega}_{R}\right]^{T}$ are the vector of absolute values of the weights. Besides, using the signal model, we calculate the relay transmit power as

$$
\mathbf{Q} \triangleq \mathbb{E}\left\{\mathbf{t}^{H} \mathbf{t}\right\}=\boldsymbol{\omega}^{H} \mathbf{D} \boldsymbol{\omega},
$$

where $\mathbf{D}=\mathbb{E}\left\{\mathbf{X}^{H} \mathbf{X}\right\}$ and $\mathbf{X}=\mathbf{D}(\mathbf{x})$. Assuming mutually independent unit variance $s_{1}, s_{2}$ random variable, and unit variance noise vector $\mathbf{z}$, matrix $\mathbf{D}$ can be expressed as

$$
\mathbf{D}=P_{1} \mathbf{A}+P_{2} \mathbf{B}+\mathbf{I}
$$

In the following section, the description of the power control and relay beamforming criteria is explained.

\section{Joint Power Allocation and Beamforming}

As mentioned, the design goal of this paper can be expressed as finding the power of the two users (user power control) and beamforming weights of the relays (relay beamforming) for a given channel realization which optimize TWRN performance metric. First of all, we should find a performance metric to formulate the problem. Total error rate of the users is considered in some papers [17]; others considered sum-rate of the two networks [18] and some others do the optimization subject to the minimum received SNRs [19].

Here, we consider the joint optimization of user power control and individual relay beamforming subject to the rates of the users and individual relay power. The latter one is the applicable condition which is not considered generally in the literature.

Using the notations in the previous section the design problem of power control and relay beamforming in TWRN with respect to individual relay power is represented as

$$
\begin{array}{ll}
\min _{\bar{\omega}, P_{1}, P_{2}} & P_{1}+P_{2}, \\
\text { s.t. } & \left(1+P_{1}\left|\alpha_{r}\right|^{2}+P_{2}\left|\beta_{r}\right|^{2}\right) \bar{\omega}_{r}^{2} \leq \rho_{r} \\
& \quad \forall r=1, \ldots, R \\
& \log _{2}\left(1+\mathrm{SNR}_{l}\right) \geq \gamma_{l}^{\prime}, \quad l=1,2 .
\end{array}
$$

The problem in (10a), (10b), and (10c) is nonconvex since there is the fractional quadratic form in the conditions. As in [20] we used Charnes-Cooper transformation to change the nonconvex form into the convex form. In the following it is explained.

3.1. Convex Optimization Approach. As mentioned earlier, we use the Charnes-Cooper transformation to change the nonconvex fractional QCQP into convex problem. Specifically, we substitute $\boldsymbol{\mu} / \sqrt{\lambda}=\overline{\boldsymbol{\omega}}$ and let $\overline{\boldsymbol{\omega}}^{T} \mathbf{h} \mathbf{h}^{T} \overline{\boldsymbol{\omega}}=1 / \lambda>0$. Thus, (6) may change into

$$
\begin{aligned}
& \mathrm{SNR}_{1}=\frac{P_{2}}{\lambda+\boldsymbol{\mu}^{T} \mathbf{A} \boldsymbol{\mu}}, \\
& \mathrm{SNR}_{2}=\frac{P_{1}}{\lambda+\boldsymbol{\mu}^{T} \mathbf{B} \boldsymbol{\mu}} .
\end{aligned}
$$

Then, considering (10c) and (11) we can limit the minimum power of each one of the users as

$$
\begin{aligned}
& P_{1} \geq \gamma_{2}\left(\lambda+\boldsymbol{\mu}^{T} \mathbf{B} \boldsymbol{\mu}\right), \\
& P_{2} \geq \gamma_{1}\left(\lambda+\boldsymbol{\mu}^{T} \mathbf{A} \boldsymbol{\mu}\right),
\end{aligned}
$$

where $\gamma_{l}=2^{\gamma_{l}^{\prime}}-1$ for $l=1,2$ is the required SNR to dictate the required rate. Accordingly, end-user's required rates limit their required power. Thus, instead of minimizing the consumed power we can minimize the lower bounds in (12). Furthermore, by new variables $\lambda$ and $\boldsymbol{\mu}$, we can change (10b) into $\boldsymbol{\mu}^{T} \ell_{r}^{T} \ell_{r} \Lambda \boldsymbol{\mu} \leq \lambda \rho_{r}$ for $r=1,2, \ldots, R$, where $\Lambda$ is 


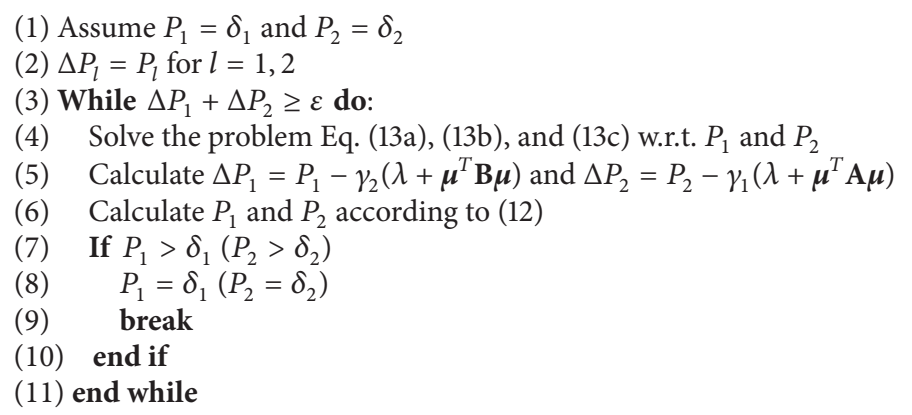

Algorithm 1: Joint power allocation and beamforming.

diagonal matrix with $\left(1+P_{1}\left|\alpha_{r}\right|^{2}+P_{2}\left|\beta_{r}\right|^{2}\right)$ for $r=1,2, \ldots, R$ as its main diagonal and $\ell_{r}$ is the zero vector except for the $r$ th entry which is one. Thus, the optimization problem equations (10a), (10b), and (10c) would be reformulated as

$$
\begin{aligned}
\min _{\boldsymbol{\mu}, \lambda, P_{1}, P_{2}} & \gamma_{1}\left(\lambda+\boldsymbol{\mu}^{T} \mathbf{A} \boldsymbol{\mu}\right)+\gamma_{2}\left(\lambda+\boldsymbol{\mu}^{T} \mathbf{B} \boldsymbol{\mu}\right) \\
\text { s.t. } & \boldsymbol{\mu}^{T} \ell_{r}^{T} \ell_{r} \Lambda \boldsymbol{\mu} \leq \lambda \rho_{r} \quad \forall r=1, \ldots, R \\
& \mathbf{h}^{T} \boldsymbol{\mu}=1 .
\end{aligned}
$$

Note that (13c) implies that $\lambda \neq 0$, since if $\lambda=0$ then $\boldsymbol{\mu}=0$ and violates (13c). On the other hand, (13b) implies that $\lambda \geq 0$. Hence, $\lambda>0$ is guaranteed.

We have proposed an algorithm to minimize the consumed power, iteratively, since the optimization problem equations (13a), (13b), and (13c) are minimized considering two categories of variables including $\boldsymbol{\mu} \& \lambda$ and $P_{1} \& P_{2}$. A step-by-step summery of the proposed algorithm is represented in Algorithm 1. The proposed algorithm is performed in iterations. Furthermore, in each iteration, the powers are assumed to be constant and the minimization problem equations (13a), (13b), and (13c) are solved with respect to constant power allocation. Then, using the new $\lambda$ and $\boldsymbol{\mu}$, the power allocations are calculated. If the stopping criteria are not met, the iterations are continued. In fact, we suppose that power allocations are constant and equal to the previous iteration. With the constant power allocation, beamforming weights are calculated such that the consumed power is minimized and sum-rate is above the predefined value.

Since $P_{1} \geq \gamma_{2}\left(\lambda+\boldsymbol{\mu}^{T} \mathbf{B} \boldsymbol{\mu}\right)$ and $P_{2} \geq \gamma_{1}\left(\lambda+\boldsymbol{\mu}^{T} \mathbf{A} \boldsymbol{\mu}\right)$, by considering $P_{1}=\delta_{1}$ and $P_{2}=\delta_{2}$, the feasible set is not violated. Thus, using the constant values for $P_{1}$ and $P_{2}$ in the feasible set of (13a), (13b), and (13c), the lower bounds of (12) are minimized. After minimizing with constant $P_{1}$ and $P_{2}$ in feasible set of (13a), (13b), and (13c), the values of $P_{1}$ and $P_{2}$ are updated using $\boldsymbol{\mu}$ and $\lambda$. This process is repeated till the optimized values are obtained using the predefined threshold for the changes of transmitted powers. As an example, we consider $P_{1 \text { opt }}$ and $P_{2 \text { opt }}$ as the optimal values and $\widetilde{P}_{1} \leq P_{1 \text { opt }}$ and $\widetilde{P}_{2} \leq P_{2 \text { opt }}$ as the new values. Consequently, $\widetilde{P}_{1} \leq \delta_{1}$ and $\widetilde{P}_{2} \leq \delta_{2}$. But if we consider (11), the amounts of $\mathrm{SNR}_{1}$ and $\mathrm{SNR}_{2}$ are decreased which is violation of condition (10c). Thus, the values of $P_{1 \text { opt }}$ and $P_{2 \text { opt }}$ are the optimal values. In this proof, one may consider that decreasing $P_{1 \text { opt }}$ and $P_{2 \text { opt }}$ will not violate condition (10c), but this is false. Since values of $P_{1}$ and $P_{2}$ are selected marginally according to (12), decreasing the marginal values will violate condition $(10 \mathrm{c})$, definitely.

The complexity of the algorithm is related to the complexity of convex optimization. Size of convex optimization problems is really critical in convex methods. In this algorithm, the size of problem is $R+1$ which is related to the number of relays. Consequently, by increasing the number of relays, complexity of the algorithm is increased. Complexity of interior points methods, which are usually used in QCQP optimization problems, is $O((R+1) \ln (1 / \varepsilon))$ [21], where $\varepsilon$ is the accuracy and $(R+1)$ is the size of problem.

Therefore, we can calculate $P_{1}, P_{2}, \lambda$, and $\boldsymbol{\mu}$ using the proposed Algorithm 1. This process is performed in the control center and the parameters for calculating the weight of each relay individually are determined. In the following section, distributed beamforming will be discussed.

\section{Distributed Power Allocation and Beamforming}

After determining the weights of relays, the control center informs each of $R$ relays about their own weights. This methodology degrades the bandwidth efficiency, especially when the number of relays is high due to the signaling contamination. Accordingly, the control center would transmit the common parameters till each of the nodes calculates its own weight with the local CSI.

Having (13a), (13b), and (13c), we can write the KarushKuhn-Tucker (KKT) [21] conditions as follows:

$$
\begin{array}{r}
2\left(\gamma_{1} \mathbf{A}+\gamma_{2} \mathbf{B}+\sum_{r} \ell_{r}^{T} \ell_{r} a_{r}^{*} \boldsymbol{\Lambda}\right) \overline{\boldsymbol{\omega}} \sqrt{\lambda}-b^{*} \mathbf{h}=0, \\
\gamma_{1}+\gamma_{2}-\sum_{r} a_{r}^{*} \rho_{r}=0, \\
\forall r \in 1,2, \ldots, R
\end{array}
$$




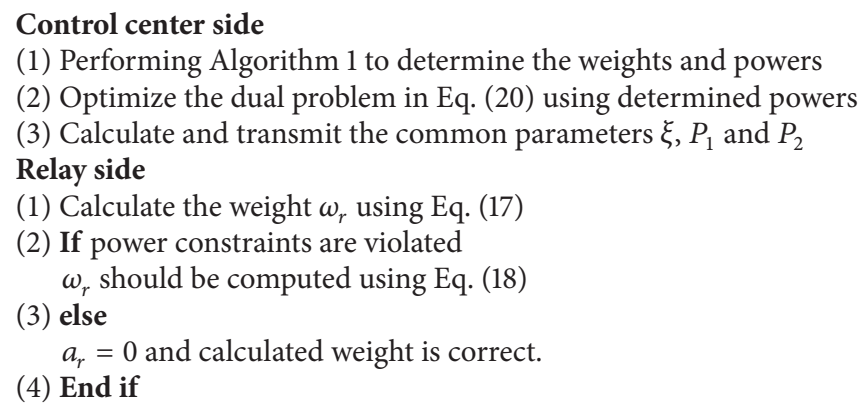

Algorithm 2: Distributed joint power allocation and beamforming.

$$
\begin{aligned}
a_{r}^{*}\left(\left(1+P_{1}\left|\alpha_{r}\right|^{2}+P_{2}\left|\beta_{r}\right|^{2}\right)\left|\bar{\omega}_{r}\right|^{2}-\lambda \rho_{r}\right) & =0, \\
\forall r & \in 1,2, \ldots, R,
\end{aligned}
$$

where $\mathbf{a}^{*}=\left[a_{1}^{*}, a_{2}^{*}, \ldots, a_{R}^{*}\right]^{T}$ and $b^{*}$ are optimal Lagrange dual variables. According to (14a) we can define weight of each relay explicitly as

$$
\overline{\boldsymbol{\omega}}=\xi\left(\gamma_{1} \mathbf{A}+\gamma_{2} \mathbf{B}+\sum_{r} \ell_{r}^{T} \ell_{r} a_{r}^{*} \boldsymbol{\Lambda}\right)^{-1} \mathbf{h}
$$

where $\xi=b^{*} / \sqrt{4 \lambda}$. Hence, the weights of each relay are determined by the following equation:

$$
\bar{\omega}_{r}=\frac{\xi h_{r}}{\gamma_{1}\left|\alpha_{r}\right|^{2}+\gamma_{2}\left|\beta_{r}\right|^{2}+a_{r}^{*}\left(1+P_{1}\left|\alpha_{r}\right|^{2}+P_{2}\left|\beta_{r}\right|^{2}\right)} .
$$

Obviously, each relay needs its own $a_{r}^{*}$ to determine the corresponding beamforming weight. Since $a_{r} \geq 0$, we consider two different cases. If $a_{r}=0$, then simply the weight $\bar{\omega}_{r}$ is

$$
\bar{\omega}_{r}=\frac{\xi h_{r}}{\gamma_{1}\left|\alpha_{r}\right|^{2}+\gamma_{2}\left|\beta_{r}\right|^{2}} .
$$

On the other hand, if $a_{r}>0$, then the complementary slackness equation (14c) holds if and only if

$$
\bar{\omega}_{r}=\sqrt{\frac{\rho_{r}}{1+P_{1}\left|\alpha_{r}\right|^{2}+P_{2}\left|\beta_{r}\right|^{2}}} .
$$

However, if $\gamma_{1}$ and $\gamma_{2}$ are determined in each relay, it can calculate its own weight using (16) and if they are not determined, this should be informed by control center. Here, we consider the determined $\gamma_{1}$ and $\gamma_{2}$. Practically, when considering some relays to exchange information, the considered rate of each user is determined for the relays, too. So, control center determines $\xi, P_{1}$, and $P_{2}$ for all the relays after performing optimization problem. For this purpose the control center may solve the Lagrange dual problem directly and determine the dual parameters as well. Therefore, we can write the Lagrange dual problem by considering the Lagrange function as

$$
\begin{aligned}
\mathscr{L}= & \gamma_{1}\left(\lambda+\boldsymbol{\mu}^{T} \mathbf{A} \boldsymbol{\mu}\right)+\gamma_{2}\left(\lambda+\boldsymbol{\mu}^{T} \mathbf{B} \boldsymbol{\mu}\right) \\
& +\sum_{r} \boldsymbol{\mu}^{T} \ell_{r}^{T} \ell_{r} a_{r} \boldsymbol{\Lambda} \boldsymbol{\mu}-\sum_{r} \lambda a_{r} \rho_{r}+b\left(\mathbf{h}^{T} \boldsymbol{\mu}-1\right) \\
= & \boldsymbol{\mu}^{T}\left(\gamma_{1} \mathbf{A}+\gamma_{2} \mathbf{B}+\sum_{r} \ell_{r}^{T} \ell_{r} a_{r} \boldsymbol{\Lambda}\right) \boldsymbol{\mu}+b \mathbf{h}^{T} \boldsymbol{\mu} \\
& +\lambda\left(\gamma_{1}+\gamma_{2}-\sum_{r} a_{r} \rho_{r}\right)-b .
\end{aligned}
$$

Consequently, the Lagrange dual problem in epigraph form by introducing $\kappa$ is formulated as

$$
\begin{array}{ll}
\min _{\mathbf{a}, b, \kappa} & \kappa \\
\text { s.t. } & \left(\begin{array}{cc}
\gamma_{1} \mathbf{A}+\gamma_{2} \mathbf{B}+\sum_{r} a_{r} \ell_{r}^{T} \ell_{r} \Lambda & \frac{b}{2} \mathbf{h} \\
\frac{b}{2} \mathbf{h}^{T} & b+\kappa
\end{array}\right) \succeq 0 \\
& \gamma_{1}+\gamma_{2}-\sum_{r} a_{r} \rho_{r}=0 \\
& \mathbf{h}^{T} \boldsymbol{\mu}=1 .
\end{array}
$$

Eventually, the distributed power allocation and beamforming are represented in Algorithm 2, step-wise. At first, the individual powers and beamforming weights are calculated using Algorithm 1 in control center. Then, (20) is optimized using resultant $P_{1}$ and $P_{2}$ and $\mathbf{a}$ and $b$ are calculated. At last, $\xi=b / \sqrt{4 \lambda}$ is calculated and $P_{1}, P_{2}$, and $\xi$ are transmitted to the relays. Each relay assumes that $a_{r}$ is equal to zero and calculates the weight using (17). Then, it checks the power constraints and if it is violated, the weight would be calculated using (18). Otherwise, the first assumption $\left(a_{r}=0\right)$ is correct.

The same as Algorithm 1, the complexity of Algorithm 2 is related to the size of problem. Here, the size of optimization problem is $(R+3)$, which implies that, by increasing the number of relays, the complexity is increased, as well. 


\section{Numerical Results}

In this section, we demonstrate the performance of our proposed beamformer. In the simulation setup, all channels are independently distributed according to $\mathscr{C} \mathscr{N}(0,1)$. We also get the average over 1000 channel realizations due to the stochastic behavior. The maximum available power for each user is considered $10 \mathrm{~dB}$.

The design criteria are based on the minimizing consumed power subject to required rates of the transceivers. As a consequence, we have calculated the required rates and consumed powers. In addition, we calculate the power efficiency of the TWRN defined as

$$
\eta \triangleq \mathbb{E}\left[\frac{R_{1}+R_{2}}{P_{1}+P_{2}+\sum_{r=1}^{R} Q_{r}}\right],
$$

where $P_{1}$ and $P_{2}$ are the end-users powers and $Q_{r}=\left(P_{1}\left|\alpha_{r}\right|^{2}+\right.$ $\left.P_{2}\left|\beta_{r}\right|^{2}+1\right)\left|\bar{\omega}_{r}\right|^{2}$ is the power of the relays. The power efficiency $(\eta)$ is the average of sum-rate of the end-users achieved in the network related to the consumed power in the whole network. It is worthy to note that the optimized solution is for the power and rates not the efficiency. In fact, we choose this power efficiency metric to compare our results with some of the previous works in the literature. To validate our results, we compare our optimal solution results with the case without any power control and beamforming called "NoPC NoBF." In case of "NoPC NoBF," the weights of the relays are calculated according to (18), where $P_{1}, P_{2}$, and $\rho_{r}$ are considered to be maximum available values.

5.1. Uniform Maximum Power. Here, we consider a uniform maximum power distribution. In other words, $\delta_{1}=\delta_{2}=\rho_{r}=$ $10(\mathrm{~W})$ for $r=1,2, \ldots, R$. Furthermore, the number of relays $R$ is assumed to be 10 . Remember that the power allocation to each node in the network is within the predefined interval using $\delta_{1}$ and $\delta_{2}$ for end-users and $\rho_{r}$ for each relay node. Actually, there are 12 nodes in the network. Furthermore, two different rate settings are considered: Scenario (1) $R_{1}=R_{2}$; Scenario (2) $R_{1}=5 R_{2}$

Figure 2 represents the consumed sum-power in both end-users and relays versus required rate in each of the endusers. In other words, sum-power is calculated as $P_{1}+P_{2}+$ $\sum_{r=1}^{R} Q_{r}$. Expectedly, the distributed proposed power control and beamforming are matched with the centralized one. As depicted, by 10 relays the consumed sum-power is increased to the maximum available power, to provide sum-rates. In this figure, two different scenarios are compared. In the second scenario, transmitting power of one of the users meets the maximum available power, though the consumed power is relatively constant for more than $6(\mathrm{bps} / \mathrm{Hz})$ sum-rates.

In Figure 3, the network power efficiency is compared. As it is obvious, by increasing the required sum-rate we can see that the power efficiency is decreased. Considering scenario (1), we can see that the power efficiency of the proposed algorithm is decreased rather than the "NoPC NoBF" case after sum-rate $=5.5(\mathrm{bps} / \mathrm{Hz})$. In this case, the TWRN could not cover the required sum-rate; hence, the efficiency is decreased by increasing the whole power.
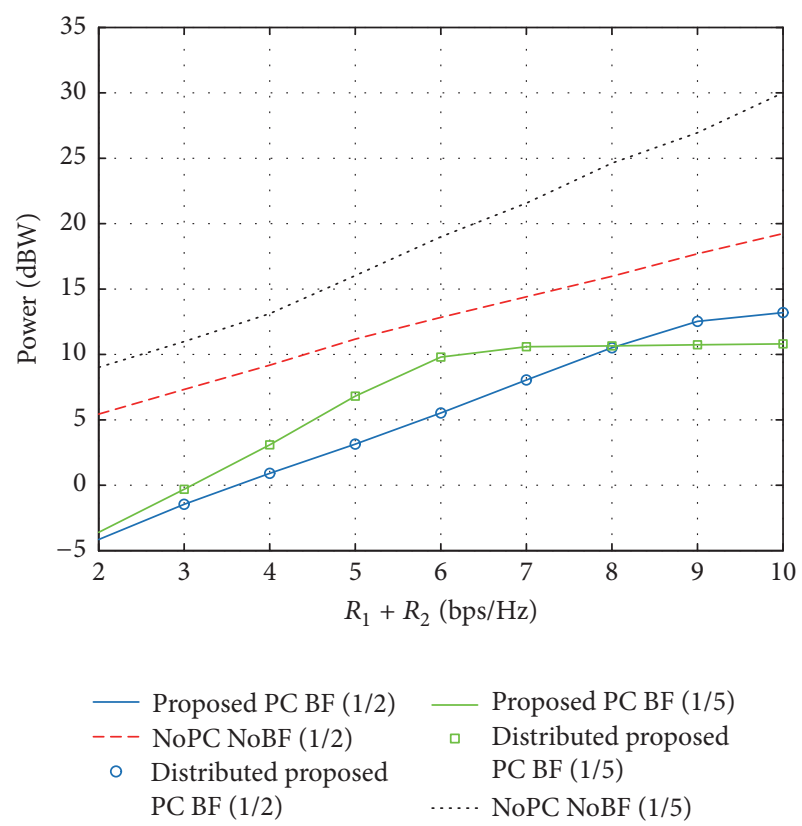

FIgURE 2: Comparison of the proposed algorithm with constant power allocation scheme from power point of view for 10 relays.

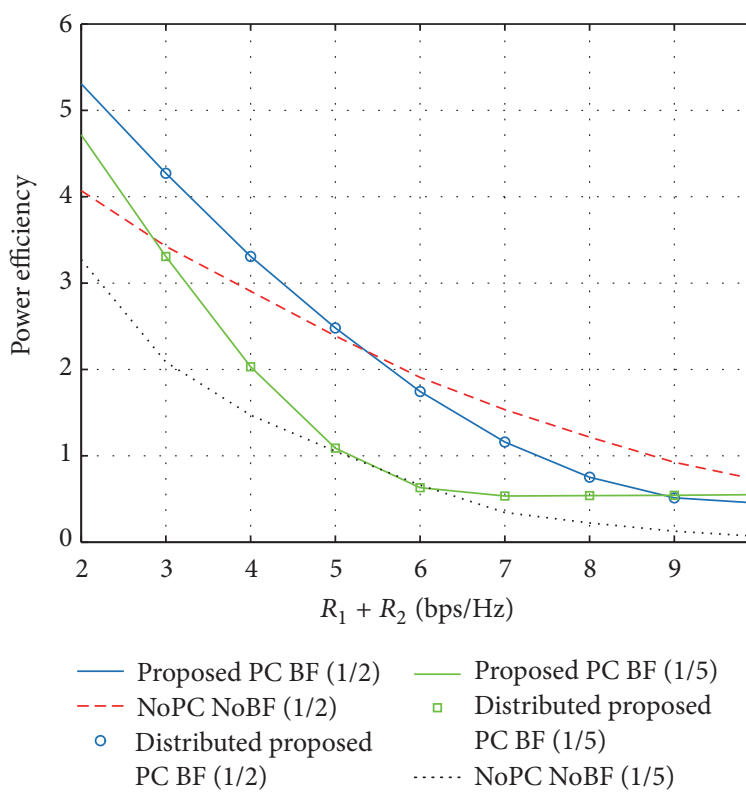

FIGURE 3: Comparison of the proposed algorithm with constant power allocation scheme from power efficiency point of view for 10 relays.

When we increase the number of relays, this phenomenon is bypassed. On the other hand, this issue is not encountered in the case of scenario (2). In fact, in this scenario the sumrate is decreased more than the previous one; therefore, the power efficiency is not decreased more than "NoPC NoBF." This figure represents that for the TWRNs supplied by few number of the relays this method is not efficient, from power efficiency point of view. In the remainder of the simulation we should consider the case of more relays. 


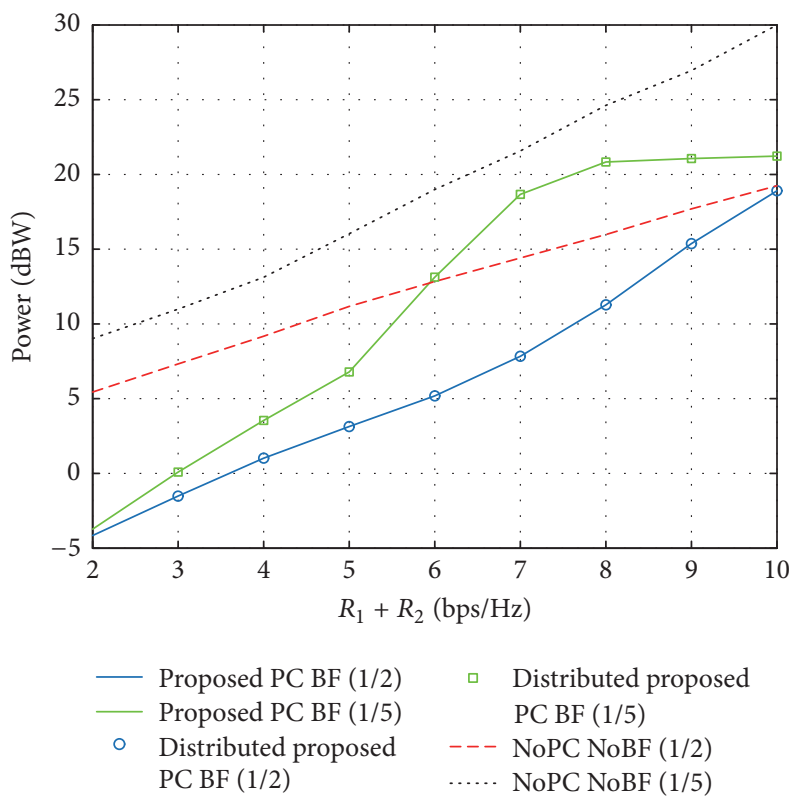

FIGURE 4: Comparison of the proposed algorithm with constant power allocation scheme from power point of view for 10 relays with nonuniform maximum power distribution.

5.2. Nonuniform Maximum Power. Here, we consider a nonuniform maximum power distribution. In other words, $\delta_{1}=\rho_{r}=10(\mathrm{~W})$ for $r=1,2, \ldots, R$ and $\delta_{2}=100(\mathrm{~W})$. Furthermore, the number of relays $R$ is assumed to be 10 . Actually, there are 12 nodes in the network. Furthermore, three different rate settings are considered: Scenario (1) $R_{1}=R_{2}$; Scenario (2) $R_{1}=5 R_{2}$; Scenario (3) $R_{2}=5 \mathrm{R}_{1}$. To represent the results, we consider scenarios (1) and (2) in one category and scenarios (1) and (3) in the other category.

Figures 4 and 5 represent the simulation results, specifically nonuniform maximum power simulation results for both scenarios (1) and (2). As depicted, the difference between Figures 3 and 5 is negligible. This is for the reason that employing 10 relays for the assumed distributed power and sum-rate is marginal. Again, we consider that maximizing the efficiency of the power was not the goal of this paper. Moreover, the power efficiency of the second scenario is worse than the case of "NoPC NoBF," which is due to the nonuniform power distribution. Besides, the consumed power simulation results in Figure 4 demonstrate that the nonincreasing behavior of the second scenario is encountered in more sum-rate.

Figures 6 and 7 represent the simulation results, specifically nonuniform maximum power simulation results for both scenarios (1) and (3). According to (11), $R_{1}$ is related to $P_{2}$ and $R_{2}$ is related to $P_{1}$. Consequently, when we limit the maximum available power by $\delta_{1}$ and define $R_{2}=5 R_{1}$, obviously, the limitation of maximum power makes it difficult for the network to satisfy $R_{2}=5 R_{1}$. This phenomenon is represented in Figures 6 and 7 explicitly. As depicted, in both figures, the result is almost identical to the case of uniform maximum power distribution. This identical

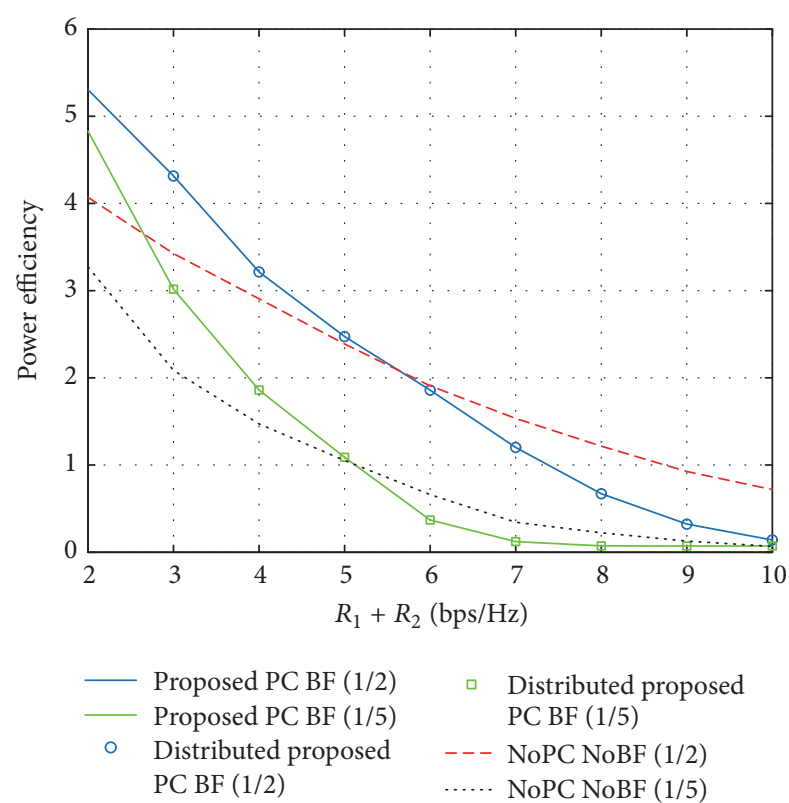

FIGURE 5: Comparison of the proposed algorithm with constant power allocation scheme from power efficiency point of view for 10 relays with nonuniform maximum power distribution.

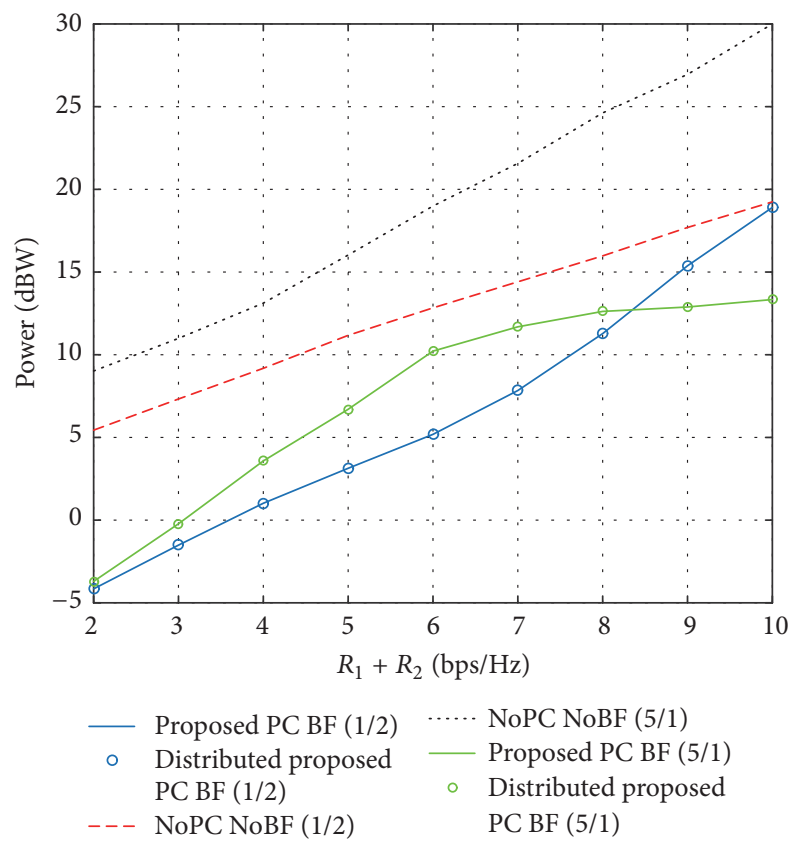

FIGURE 6: Comparison of the proposed algorithm with constant power allocation scheme from power point of view for 10 relays with nonuniform maximum power distribution.

behavior validates the limitation of maximum power which was encountered in uniform maximum power in Figure 3.

5.3. Impact of Relays. In this section, the impact of relay numbers is studied on the defined metrics. In order to represent the impact of the relay numbers on the proposed 


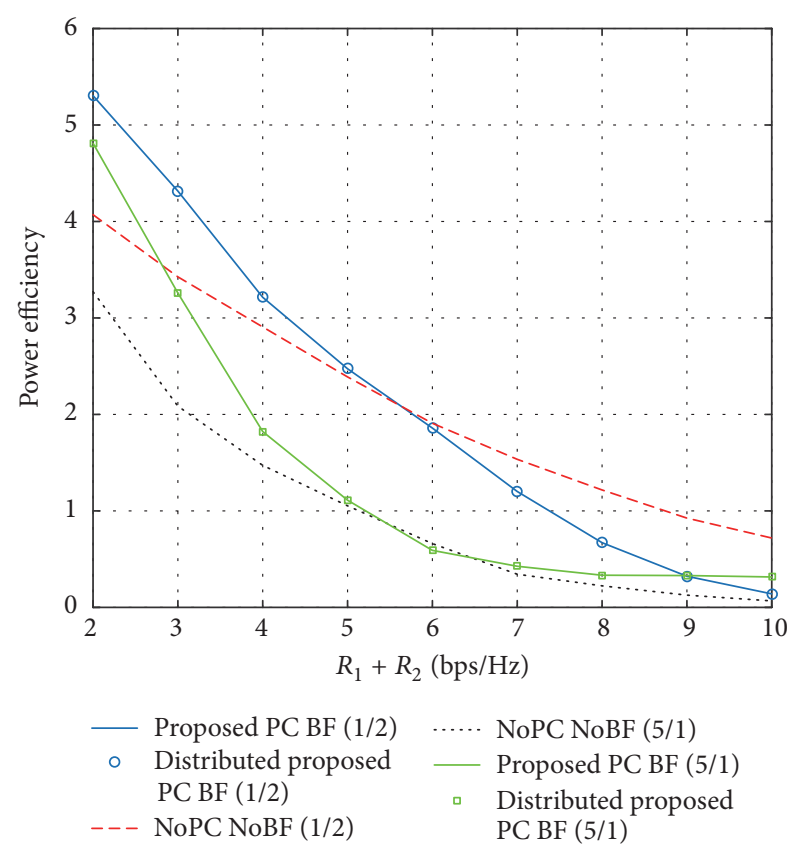

Figure 7: Comparison of the proposed algorithm with constant power allocation scheme from power efficiency point of view for 10 relays with nonuniform maximum power distribution.

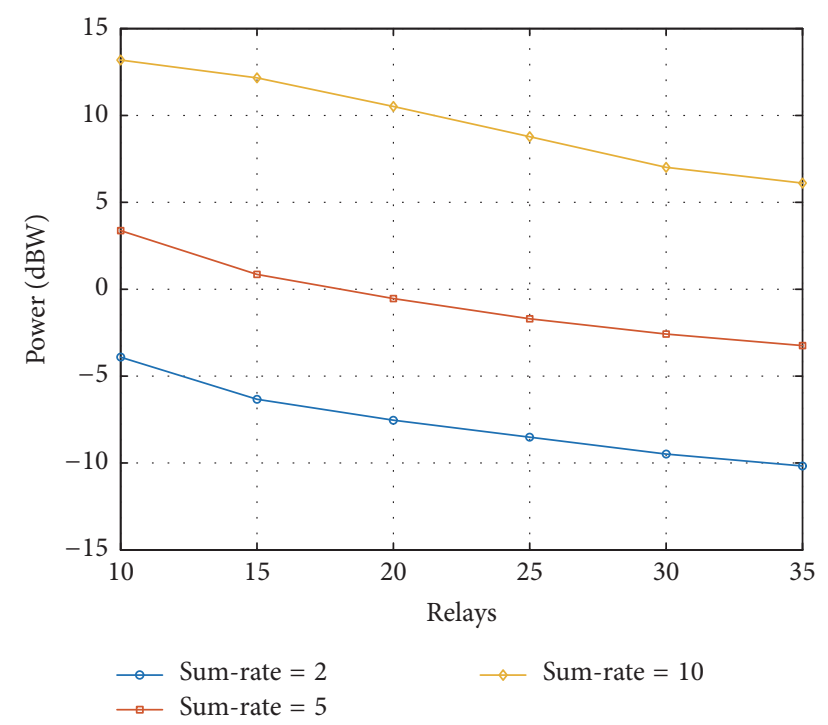

FIgURE 8: Comparison of the proposed algorithm with constant power allocation scheme from power point of view for different number of relays.

power control and beamforming, we consider two different scenarios: Scenario (1) $R_{1}=R_{2}$; Scenario (2) $R_{1}=5 R_{2}$. Furthermore, for simplicity just two different sum-rates are considered. For each of the scenarios, the number of relays in the TWRN is changed from 10 to 30 . Besides, maximum power is distributed uniformly; that is, $\delta_{1}=\delta_{2}=\rho_{r}=10(\mathrm{~W})$ for $r=1,2, \ldots, R$. As demonstrated in Figure 8 , increasing the number of relays would decrease the consumed power in

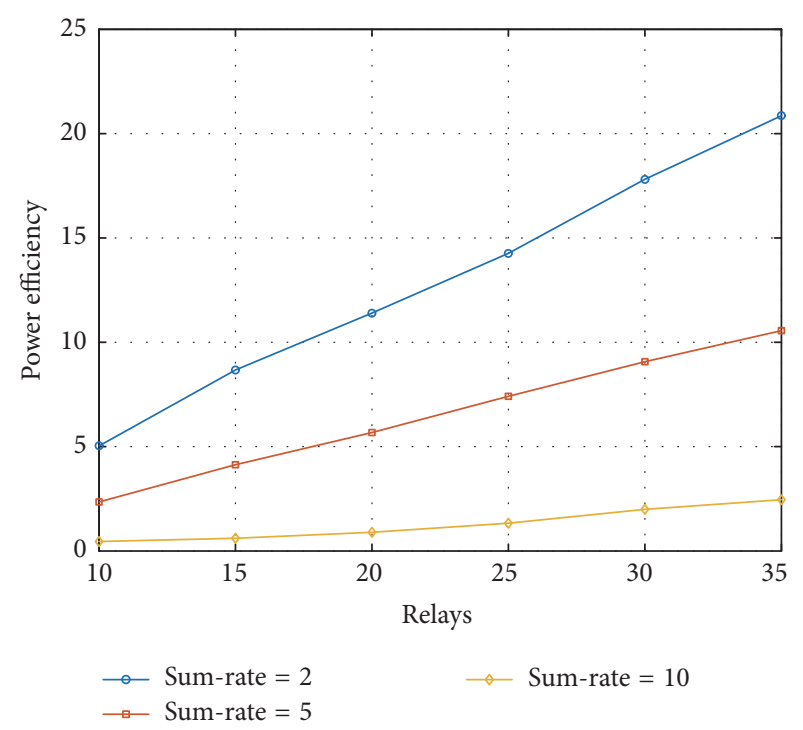

FIgURE 9: Comparison of the proposed algorithm with constant power allocation scheme from power efficiency point of view for different number of relays.

the network which is expected. On the other hand, increasing the number of relays would increase the power efficiency according to Figure 9. These two consequences are occurred in each of the sum-rates but with a little different impact. In other words, the impact of relay increasing on the power decreasing is more in the sum-rate of 2 rather than sum-rate of 10 . In other words, the power consumption is more affected by increasing the number of relays in lower sum-rates.

\section{Conclusion}

In this paper, we have proposed a beamforming and power allocation algorithm based on minimizing the power with respect to end-user available rates. Actually, we modelled it by a power allocation problem and then tried to solve the optimization problem by convex programming. For the sake of using convex programming, we have applied CharnesCooper transformation and SDP relaxation to the optimization problem. Furthermore, we considered this problem constrained to a limited source of energy for each node in TWRN which is a practical assumption. Eventually, a distributed version of the proposed algorithm was represented which determines each of the weights and power individually by just some common parameters. Besides, common parameters were determined and transmitted by a control center in the network. Moreover, to represent the efficiency of the proposed method, we introduced an efficiency metric $\eta$ which demonstrated the sum-rate to the consumed sumpower. Expectedly, this metric was increased by increasing the number of utilized relays.

\section{Conflicts of Interest}

The authors declare that there are no conflicts of interest regarding the publication of this paper. 


\section{References}

[1] T. M. Cover and A. El Gamal, "Capacity theorems for the relay channel," Institute of Electrical and Electronics Engineers Transactions on Information Theory, vol. 25, no. 5, pp. 572-584, 1979.

[2] J. N. Laneman, D. Tse, and G. W. Wornell, "Cooperative diversity in wireless networks: efficient protocols and outage behavior," Institute of Electrical and Electronics Engineers Transactions on Information Theory, vol. 50, no. 12, pp. 3062-3080, 2004.

[3] E. Telatar, "Capacity of multi-antenna Gaussian channels," European Transactions on Telecommunications, vol.10, no. 6, pp. 585-595, 1999.

[4] A. Goldsmith, S. A. Jafar, N. Jindal, and S. Vishwanath, "Capacity limits of MIMO channels," IEEE Journal on Selected Areas in Communications, vol. 21, no. 5, pp. 684-702, 2003.

[5] M. Zaeri-Amirani, S. Shahbazpanahi, T. Mirfakhraie, and K. Ozdemir, "Performance tradeoffs in amplify-and-forward bidirectional network beamforming," IEEE Transactions on Signal Processing, vol. 60, no. 8, pp. 4196-4209, 2012.

[6] H. Chen, S. Shahbazpanahi, and A. B. Gershman, "Filter-andforward distributed beamforming for two-way relay networks with frequency selective channels," IEEE Transactions on Signal Processing, vol. 60, no. 4, pp. 1927-1941, 2012.

[7] R. Vahidnia and S. Shahbazpanahi, "Pre-channel equalization and distributed beamforming in asynchronous single-carrier bi-directional relay networks," IEEE Transactions on Signal Processing, vol. 64, no. 4, pp. 1907-1915, 2016.

[8] Y. Jing and S. ShahbazPanahi, "Max-min optimal joint power control and distributed beamforming for two-way relay networks under per-node power constraints," IEEE Transactions on Signal Processing, vol. 60, no. 12, pp. 6576-6589, 2012.

[9] S. Shahbazpanahi, "A semi-closed-form solution to optimal decentralized beamforming for two-way relay networks," in Proceedings of the 3rd IEEE International Workshop on Computational Advances in Multi-Sensor Adaptive Processing, (CAMSAP '09), pp. 101-104, IEEE, Aruba, Dutch Antilles, Netherlands, December 2009.

[10] S. Shahbazpanahi and M. Dong, "A semi-closed form solution to the SNR balancing problem of two-way relay network beamforming," in Proceedings of the IEEE International Conference on Acoustics, Speech, and Signal Processing, (ICASSP '10), pp. 25142517, IEEE, Dallas, TX, USA, March 2010.

[11] S. Talwar, Y. Jing, and S. Shahbazpanahi, "Joint relay selection and power allocation for two-way relay networks," IEEE Signal Processing Letters, vol. 18, no. 2, pp. 91-94, 2011.

[12] S. Shahbazpanahi and M. Dong, "Achievable rate region and sum-rate maximization for network beamforming for bidirectional relay networks," in Proceedings of the IEEE International Conference on Acoustics, Speech, and Signal Processing, (ICASSP '10), pp. 2510-2513, IEEE, Dallas, TX, USA, March 2010.

[13] R. Vaze and R. W. Heath Jr., "Optimal amplify and forward strategy for two-way relay channel with multiple relays," in Proceedings of the IEEE Information Theory Workshop on Networking and Information Theory, (ITW '09), pp. 181-185, IEEE, Volos, Greece, June 2009.

[14] A. De Maio, Y. Huang, D. P. Palomar, S. Zhang, and A. Farina, "Fractional QCQP with applications in ML steering direction estimation for radar detection," IEEE Transactions on Signal Processing, vol. 59, no. 1, pp. 172-185, 2011.
[15] C. Jeong, I.-M. Kim, and D. I. Kim, "Joint secure beamforming design at the source and the relay for an amplify-and-forward MIMO untrusted relay system," IEEE Transactions on Signal Processing, vol. 60, no. 1, pp. 310-325, 2012.

[16] A. Charnes and W. W. Cooper, "Programming with linear fractional functionals," Naval Research Logistics Quarterly, vol. 9, pp. 181-186, 1962.

[17] Y. Li, R. Louie, and B. Vucetic, "Relay selection with network coding in two-way relay channels," IEEE Transactions on Vehicular Technology, vol. 59, no. 8, pp. 4489-4499, 2010.

[18] T. J. Oechtering and H. Boche, "Bidirectional regenerative halfduplex relaying using relay selection," IEEE Transactions on Wireless Communications, vol. 7, no. 5, pp. 1879-1888, 2008.

[19] I. Krikidis, "Relay selection for two-way relay channels with MABC DF: a diversity perspective," IEEE Transactions on Vehicular Technology, vol. 59, no. 10, pp. 4620-4628, 2010.

[20] Q. Li, Q. Zhang, and J. Qin, "A special class of fractional QCQP and its applications on cognitive collaborative beamforming," IEEE Transactions on Signal Processing, vol. 62, no. 8, pp. 21512164, 2014.

[21] S. Boyd and L. Vandenberghe, Convex Optimization, Cambridge University Press, 2004. 


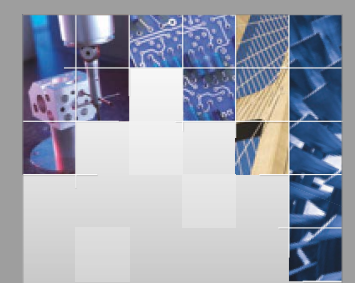

\section{Enfincering}
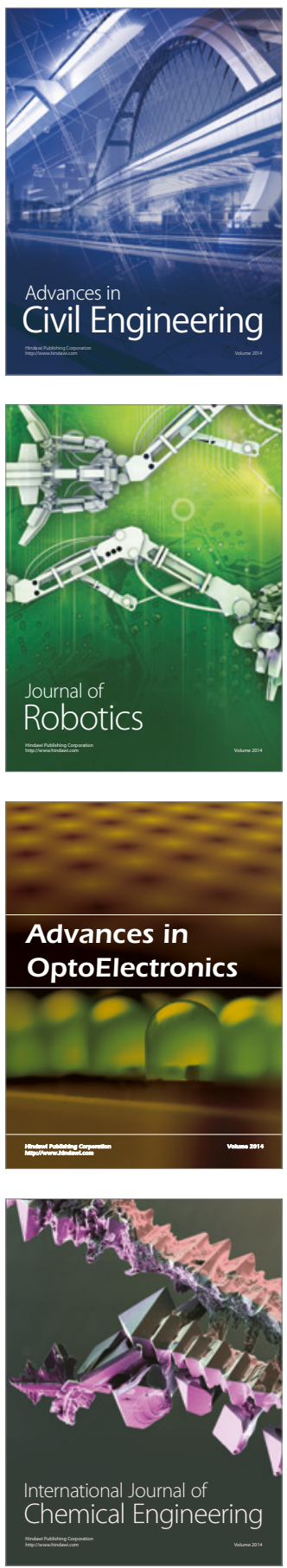

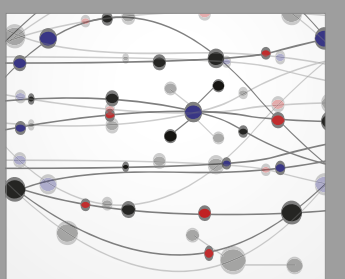

The Scientific World Journal

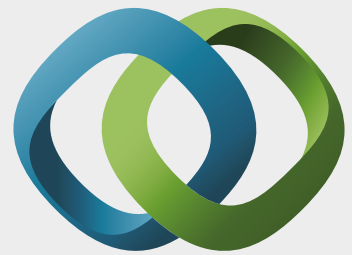

\section{Hindawi}

Submit your manuscripts at

https://www.hindawi.com
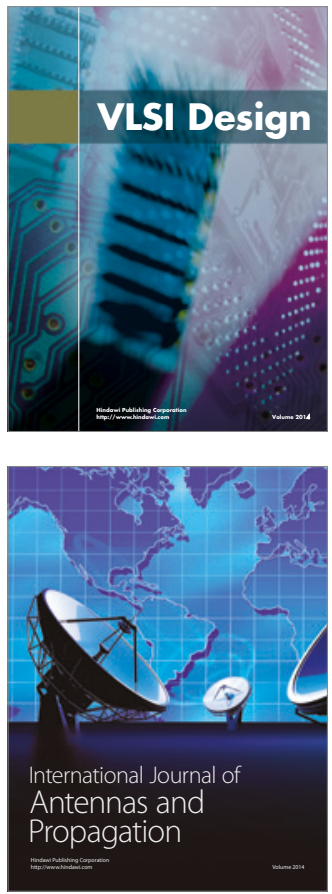

\section{Rotating}

Machinery
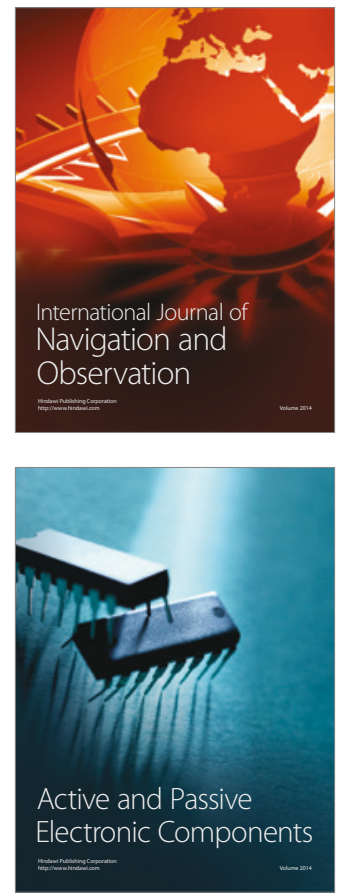
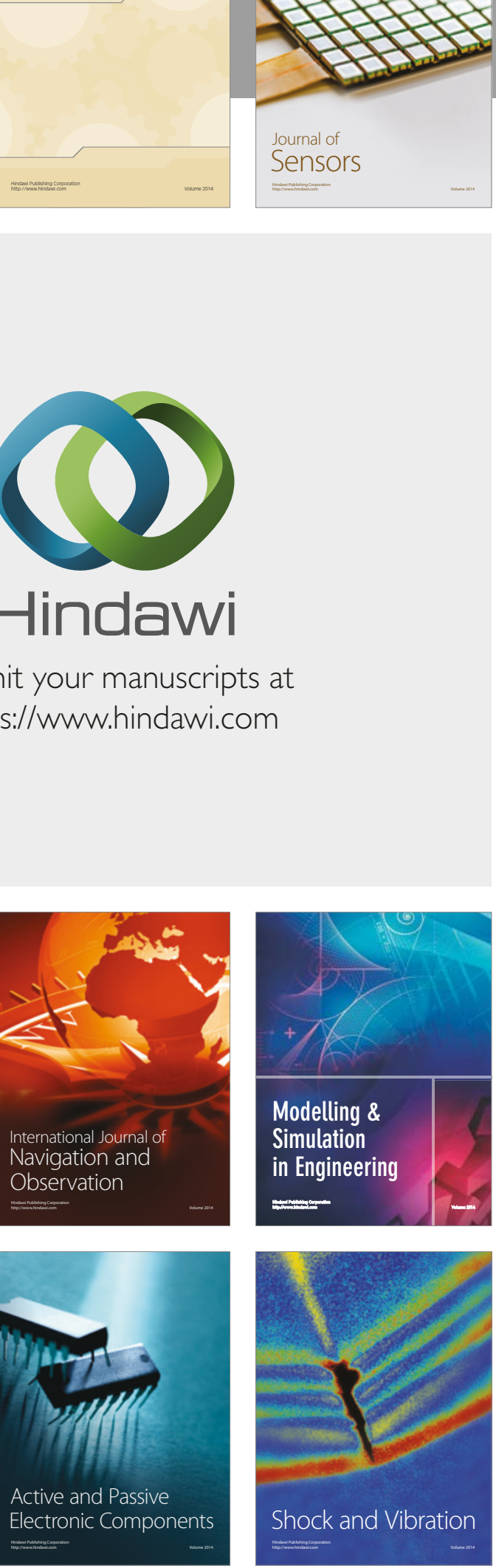
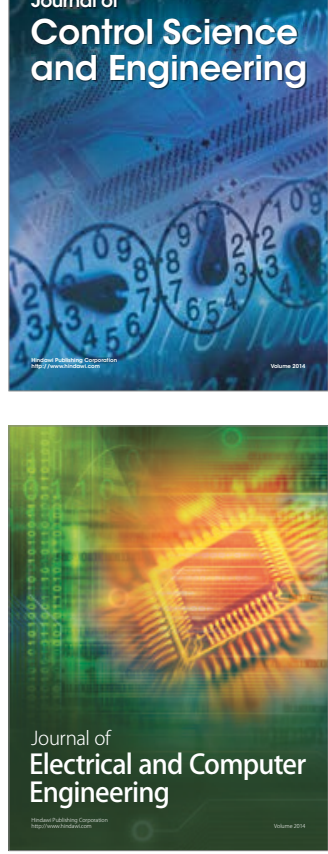

Distributed

Journal of

Control Science

and Engineering
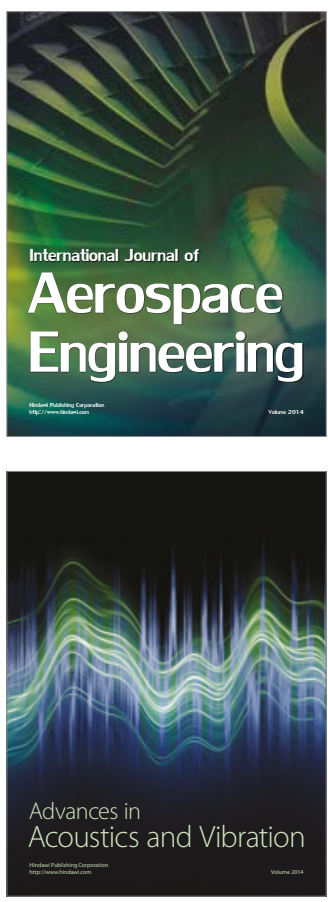

Sensor Networks 